\title{
Frequency comb generation in continuously pumped optical parametric oscillator
}

\author{
S. Mosca ${ }^{1}$, M. Parisi ${ }^{1}$, I. Ricciardi ${ }^{1}$, F. Leo ${ }^{2}$, T. Hansson ${ }^{3}$, M. Erkintalo ${ }^{4}$, P. Maddaloni ${ }^{1}$, \\ P. De Natale ${ }^{5}$, S. Wabnitz ${ }^{6,1}$, M. De Rosa ${ }^{1}$ \\ ${ }^{1}$ CNR-INO, Istituto Nazionale di Ottica, Via Campi Flegrei 34, 80078 Pozzuoli (NA), Italy \\ ${ }^{2}$ OPERA-photonics, Université Libre de Bruxelles, 50 Avenue F. D. Roosevelt, CP 194/5, B-1050 Bruxelles, Belgium \\ ${ }^{3}$ INRS-EMT, 1650 Blvd. Lionel-Boulet, Varennes, Québec J3X 1S2, Canada \\ ${ }^{4}$ The Dodd-Walls Centre for Photonic and Quantum Technologies, Department of Physics, The University of \\ Auckland, Auckland 1142, New Zealand \\ ${ }^{5}$ CNR-INO, Istituto Nazionale di Ottica, Largo E. Fermi, 50125 Firenze, Italy \\ ${ }^{6}$ Dipartimento di Ingegneria dell'Informazione, Università di Brescia, via Branze 38, 25123 Brescia, Italy \\ maurizio.derosa@ino.it
}

\begin{abstract}
We experimentally demonstrate that optical frequency combs can be generated in a cw-pumped, nearly degenerate, doubly resonant optical parametric oscillator (OPO). Moreover, we present a time-domain theoretical model of the OPO and derive a non-instantaneous mean field dynamic equation for the parametric field. Numerical simulations of the mean field equation are in good agreement with the observed comb patterns.
\end{abstract}

OCIS codes: $190.4410,190.4223$.

In order to generate optical frequency combs (OFCs), the use of passive nonlinear cavities has been shown to represent an attractive alternative to traditional techniques based on femtosecond mode-locked lasers. Indeed, comb generation has been demonstrated to occur in continuosly pumped resonators with third- or second-order nonlinearities $[1,2]$. In cavities dominated by the second-order nonlinerity, OFCs can be generated when the nonlinear crystal is phase-matched for second harmonic generation and the cavity is pumped above the threshold of the so-called internally-pumped optical parametric oscillation [2,3]. Here, we experimentally demonstrate, for the first time to the best of our knowledge, that quadratic frequency combs can be directly generated in a cw-pumped, nearly degenerate, doubly resonant optical parametric oscillator (OPO). Comb emission is observed both around the pump frequency $2 \omega_{0}$, as well as around the parametric spectral signals around $\omega_{0}$. We also report on a time-domain mean field equation for the parametric field dynamics, which includes the effect of dispersion, and thus permits for the efficient modelling of the full comb dynamics. Our equation not only gives a deep insight into the physics of comb generation, but also provides a tool to explore the comb dynamics through numerical simulation.

The OPO cavity used in our experiments is made by a 15-mm-long, periodically poled, lithium niobate crystal placed inside a four-mirrors bow-tie cavity which resonates only for the (nearly) degenerate signal-idler waves around $1064 \mathrm{~nm}\left(\omega_{0}\right)$. Two curved and one plane mirrors are highly reflecting $(99.9 \%)$, whilst the fourth outcoupling mirror has a reflectivity of $98 \%$. The OPO is pumped by a frequency doubled Nd:YAG laser up to about $1 \mathrm{~W}$ of green power at $532 \mathrm{~nm}\left(2 \omega_{0}\right)$. The power threshold for parametric oscillations is about $30 \mathrm{~mW}$. The temperature of each crystal is actively stabilized by a Peltier element. Moreover, each cavity has a mirror mounted on a piezoelectric actuator for cavity length control. The OPO cavity is locked to the laser degeneracy frequency by FM locking technique, thanks to a few-milliwatts laser beam that is phase modulated and coupled to the OPO cavity in the opposite direction of the pump beam. The infrared cavity output is sent to an optical spectrum analyzer (OSA). Fast photodetectors are used for detecting intermodal beat notes.

We adjust the crystal temperature such that the fundamental and parametric field wave vectors, $k_{2}$ and $k_{1}$, respectively, satisfy the phase matching condition $\Delta k=2 k_{1}-k_{2}=0$, corresponding to a degenerate parametric emission. We observed frequency comb emission for pump power greater than $80 \mathrm{~mW}$, when the cavity is locked resonant to the pump laser frequency $\omega_{0}$. We also explored the different dynamic regimes which occur when the cavity is detuned with respect to the laser frequency, by adding a finite offset voltage to the locking error signal. Figure 1 shows three spectra corresponding to three different detunings, from negative to positive, when the cavity is pumped by $300 \mathrm{~mW}$ of green light. For these spectra, no intermodal beat note has been detected in the $2 \mathrm{GHz}$ bandwidth of the detectors, indicating a line spacing larger than the cavity free spectral range (505 MHz). Indeed, the spectra of Fig. 1(a) and (b) display a resolved comb structure made of lines separated by about $20 \mathrm{GHz}(\mathrm{RBW}=3 \mathrm{GHz})$. 

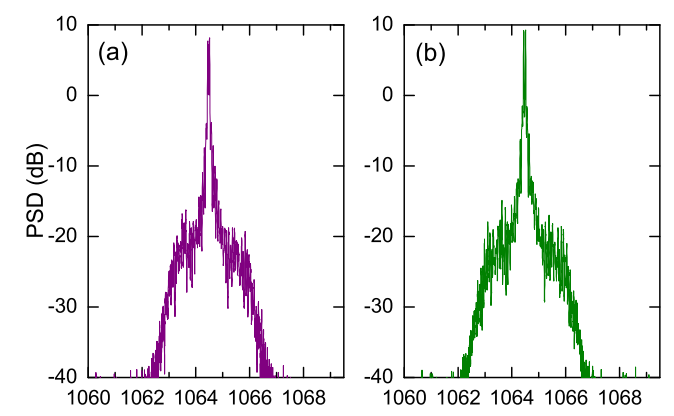

Wavelength $(\mathrm{nm})$
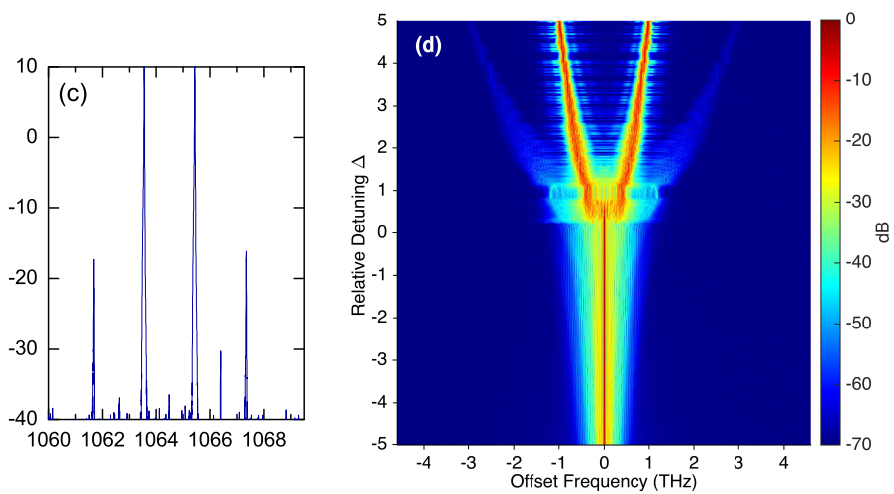

Fig. 1. (a)-(c) Experimentally measured spectral emission of the OPO for different values of the cavity detuning $\Delta=\delta_{1} / \alpha_{1}=-0.3,0.0,+0.3$, respectively, with $300 \mathrm{~mW}$ of pump power $(\mathrm{RBW}=3 \mathrm{GHz})$. (d) Numerically simulated comb spectra as the cavity detuning is swept across a resonance.

Starting from the coupled mode equations that describe the propagation of the amplitude envelopes of the pump and parametric fields, along with the boundary condition for the fields at the end of each round trip [4], we derived the following mean field equation for the parametric field amplitude $A$

$$
t_{\mathrm{R}} \frac{\partial A(t, \tau)}{\partial t}=\left(-\alpha_{1}-i \delta_{1}-i \frac{L k_{1}^{\prime \prime}}{2} \frac{\partial^{2}}{\partial \tau^{2}}\right) A-\mu^{2} A^{*}\left[A^{2}(t, \tau) \otimes I(\tau)\right]+i \mu B_{\text {in }} A^{*},
$$

where $t$ is the "slow time" variable linked to the roundtrip index and $\tau$ is the "fast" time in the frame of reference moving at the group velocity of the parametric frequency; $t_{R}$ is the cavity round-trip time; $\alpha_{1}$ are the cavity total losses; $\delta_{1}$ is the laser to cavity detuning; $L$ is the cavity length; $k_{1}^{\prime \prime}$ is the GVD at the parametric frequency; $\mu$ is the nonlinear coupling constant; and $B_{\text {in }}$ is the pump beam field amplitude. The symbol $\otimes$ denotes convolution and $I(\tau)$ is the same delayed response already derived for the comb dynamics in singly resonant cavity SHG $[4,5]$. Stability analysis of Eq. (1) is not trivial, because of the convolution term. However, the equation can be numerically integrated to investigate the dynamics for a wide range of experimental parameters. Figure 1(d) shows a numerical simulation of the parametric spectra while varying the detuning from negative to positive.

As a major result, our findings path the way to direct generation of OFCs at longer wavelength starting from $\mathrm{cw}$ lasers at shorter wavelength, of great interest, e.g., for extending OFCs in the mid and far infrared, with remarkable application from astronomy to molecular spectroscopy.

Work supported by Ministero dell'Istruzione, dell'Università e della Ricerca (Projects: FIRB-RBFR13QUVI “Optomechanical tailoring of squeezed light"; and PRIN-2015KEZNYM, "NEMO-Nonlinear dynamics of optical frequency combs").

\section{References}

1. P. Del'Haye, A. Schliesser, O. Arcizet, T. Wilken, R. Holzwarth, and T. J. Kippenberg, “Optical frequency comb generation from a monolithic microresonator," Nature 450, 1214-1217 (2007).

2. I. Ricciardi, S. Mosca, M. Parisi, P. Maddaloni, L. Santamaria, P. De Natale, and M. De Rosa, "Frequency comb generation in quadratic nonlinear media," Phys. Rev. A 91, 063839 (2015).

3. S. Mosca, I. Ricciardi, M. Parisi, P. Maddaloni, L. Santamaria, P. De Natale, and M. De Rosa, "Direct generation of optical frequency combs in $\chi^{(2)}$ nonlinear cavities ," Nanophotonics 5, 316-331 (2016).

4. F. Leo, T. Hansson, I. Ricciardi, M. De Rosa, S. Coen, S. Wabnitz, and M. Erkintalo, "Walk-Off-Induced Modulation Instability, Temporal Pattern Formation, and Frequency Comb Generation in Cavity-Enhanced SecondHarmonic Generation,” Phys. Rev. Lett. 116, 033901 (2016).

5. T. Hansson, F. Leo, M. Erkintalo, S. Coen, I. Ricciardi, M. De Rosa, and S. Wabnitz, "Singly resonant secondharmonic-generation frequency combs," Phys. Rev. A 95, 013805 (2017). 